\title{
Synthesis of an Azaphosphatriptycene and Its Rhodium Carbonyl Complex
}

\author{
Yu Cao ${ }^{\dagger}$ Jonathan W. Napoline, ${ }^{\dagger}, \S$ John Bacsa, ${ }^{\ddagger}$ Pamela Pollet ${ }^{\dagger}$ Jake D. Soper, ${ }^{\dagger}$ and Joseph P. Sadighi*,† \\ †School of Chemistry \& Biochemistry, Georgia Institute of Technology, Atlanta, Georgia 30332-0400, United States \\ ${ }^{\S}$ Current address: Department of Chemistry, Saint Anselm College, Manchester, New Hampshire 03102 \\ ^X-ray Crystallography Center, Department of Chemistry, Emory University, 1515 Dickey Drive, Atlanta Georgia 30322, United States
}

joseph.sadighi@chemistry.gatech.edu

Supporting Information

\section{Table of Contents}

1. General Considerations $\quad$ S-2

2. Synthesis of Azaphosphatriptycene $\mathbf{1} \quad$ S-2

3. Preparation of Complex 2, [(1)Rh(acac)(CO)] S-3

4. $\quad$ Selected Spectra $\quad$ S-3

5. General Procedure for Hydroformylation $\quad$ S-6

$\begin{array}{ll}\text { 6. Hydroformylation of 1-Octene } & \text { S-7 }\end{array}$

7. X-ray Diffraction Studies of 2, [(1)Rh(acac)(CO)] S-7

8. References $\quad$ S-11 


\section{General Considerations}

Unless otherwise indicated, manipulations were carried out in resealable glassware on a Schlenk line under an atmosphere of argon, or in an MBraun glovebox under an atmosphere of nitrogen. Glassware and magnetic stir bars were dried in a ventilated oven at $160{ }^{\circ} \mathrm{C}$ and allowed to cool under vacuum. Tetrahydrofuran (THF, EMD Millipore OmniSolv), toluene (EMD Millipore OmniSolv) and dichloromethane (EMD Millipore OmniSolv) were sparged with ultra-high purity argon (NexAir) for 45 minutes prior to first use and dried using an MBraun solvent purification system. THF and toluene were further dried over sodium benzophenone ketyl, degassed by successive freeze-pump-thaw cycles and transferred under vacuum to an oven-dried resealable flasks. Dichloromethane was further dried by stirring overnight with calcium hydride (Alfa Aesar, coarse powder), degassed by successive freezepump-thaw cycles, and transferred under vacuum into oven-dried resealable Schlenk flask. Benzene- $d_{6}$ (Cambridge Isotope Laboratories) was dried over sodium benzophenone ketyl, degassed by successive freeze-pump-thaw cycles and transferred under vacuum into oven-dried resealable flask. 1-Octene (Acros Organics), cyclohexene (Alfa Aesar), 2,3-dihydrofuran (Alfa Aesar) and 3,4-dihydro-2H-pyran (Aldrich) were dried by stirring overnight with calcium hydride (Alfa Aesar, coarse powder), degassed by successive freeze-pump-thaw cycles, and transferred under vacuum into oven-dried resealable Schlenk flasks. Anhydrous pentane (EMD Millipore Drisolv, sealed under a nitrogen atmosphere) and (acetylacetonato)dicarbonyl-rhodium(I) (Aldrich) were used as received and stored in a glovebox. Ethyl Acetate (BDH), methanol (BDH), ethanol (200 proof, Koptec), chloroform- $d$ (Cambridge Isotope Laboratories), tert-butyllithium (1.7 M in pentane, Aldrich), nitric acid (BDH, 68-70\%, ACS grade), tris(2,4-di-tertbutylphenyl)phosphite (Strem Chemicals), anhydrous magnesium sulfate (Alfa Aesar, powder), sodium hydroxide (EMD Millipore), nonanal (Aldrich, analytical standard), cyclohexanecarboxaldehyde (Aldrich), di-(4-tertbutylphenyl)amine (TCI America), tris(dibenzylideneacetone)dipalladium(0) (Aldrich), tris(o-tolyl)phosphine (Aldrich), sodium tert-butoxide (TCI America), 1-bromo-4-tert-butylbenzene (Oakwood Chemicals), $N$ bromosuccinimide (Aldrich), sodium metal (Alfa Aesar), benzophenone (Alfa Aesar), calcium hydride (Alfa Aesar), nitrogen (NexAir), syngas (CO/ $\mathrm{H}_{2}=1: 1$, NexAir) and argon (both industrial and ultra-high purity grades, NexAir) were used as received. Tap water was purified in a Barnstead International automated still prior to use.

Tris(4-tert-butylphenyl)amine ${ }^{1}$ and tris(2-bromo-4-tert-butylphenyl)amine ${ }^{2}$ were prepared according to reported procedures, and characterized by ${ }^{1} \mathrm{H}$ and ${ }^{13} \mathrm{C}$ NMR spectroscopy.

${ }^{1} \mathrm{H},{ }^{13} \mathrm{C}$ and ${ }^{31} \mathrm{P}$ NMR spectra were obtained using a Varian Mercury Vx 400 spectrometer. ${ }^{1} \mathrm{H}$ and ${ }^{13} \mathrm{C}$ NMR chemical shifts were referenced with respect to solvent signals and reported relative to tetramethylsilane. ${ }^{31} \mathrm{P}$ NMR chemical shifts were reported with $85 \%$ aqueous $\mathrm{H}_{3} \mathrm{PO}_{4}$ as external standard. Chemical shifts $\delta$ are reported in ppm. Fourier Transform Infrared spectra were collected using microcrystalline samples on a Shimadzu IRAffinity-1S Fourier transform infrared spectrophotometer. Samples were exposed to air as briefly as possible prior to data collection. Only characteristic peaks were reported in $\mathrm{cm}^{-1}$. EI-MS and GC-MS were recorded on MicroMass AutoSpec $\mathrm{M}$ by Georgia Institute of Technology mass spec facility. Elemental analyses were performed by Atlantic Microlab, Inc. in Norcross, GA.

Hydroformylation reactions were performed in a 300-mL stainless steel stirred Parr reactor (Parr Instrument Company, model 452HC, maximum allowable working pressure: 2000 psi (137.9 bar) at 350 ${ }^{\circ} \mathrm{C}$ ). The $300-\mathrm{mL}$ stainless steel Parr reactor was thoroughly washed with nitric acid (1 M, 3 x $300 \mathrm{~mL}$ ), neutralized with $1 \mathrm{M}$ NaOH solution, followed by several rinse cycles with distilled water and acetone. The reactor was dried in pieces in a ventilated oven at $160^{\circ} \mathrm{C}$ for $12 \mathrm{~h}$, then reconstructed and purged with 7 bar nitrogen 10 times with heating to $140{ }^{\circ} \mathrm{C}$. The reactor was cooled to room temperature under $\mathrm{N}_{2}$ (7 bar) before each experiment. Syngas was added to the vessel via high pressure stainless steel tubing, using a Matheson Tri-Gas control system (PAN-5500 Series Analytical Grade Panel) and 500D Syringe Pump (Teledyne Isco). Gas chromatography-flame ionization detection (GC-FID) analyses were performed using a Shimadzu GC-2010 Plus gas chromatograph fitted with a Supelco PTA-5 (30 m x $0.25 \mathrm{~mm} \times 0.50 \mu \mathrm{m}$, length $\mathrm{x}$ inside diameter x film thickness) capillary GC column. Calibration curves for 1-octene, nonanal, cyclohexene, cyclohexanecarboxaldehyde, 2,3-dihydrofuran and 3,4-dihydro-2H-pyran were recorded on the GC-FID.

\section{Synthesis of Azaphosphatriptycene 1}

Tris(2-bromo-4-tert-butylphenyl)amine $24.056 \mathrm{~g}, 6.237 \mathrm{mmol}$ ) was dissolved in THF (150 mL) in a 500-mL Schlenk flask and the solution was cooled to $-78^{\circ} \mathrm{C}$. tert-Butyllithium (1.7 $\mathrm{M}$ in pentane, $22.0 \mathrm{~mL}, 37.4 \mathrm{mmol}$ ) was added dropwise at $-78{ }^{\circ} \mathrm{C}$ and the resulting solution was stirred at $-78{ }^{\circ} \mathrm{C}$ for $2 \mathrm{~h}$. A solution of tris(2,4-di-tertbutylphenyl) phosphite (4.035 g, $6.237 \mathrm{mmol})$ in THF $(20 \mathrm{~mL})$ was added dropwise to the reaction mixture, which was allowed to warm slowly to ambient temperature, then refluxed for $96 \mathrm{~h}$. The solution was cooled to room temperature, quenched with distilled water, then extracted with ethyl acetate ( 3 portions, $50 \mathrm{~mL}$ each). The combined organic portions were washed with distilled water and dried over $\mathrm{MgSO}_{4}$. The resulting mixture was filtered, then 
concentrated in vacuo. Addition of methanol $(50 \mathrm{~mL})$ to the residue caused a microcrystalline solid to form. The offwhite precipitate was collected by filtration and recrystallized from $\mathrm{CH}_{2} \mathrm{Cl}_{2} / \mathrm{EtOH}$ to afford 1 as a white solid, $1.945 \mathrm{~g}$ (71\%). ${ }^{1} \mathrm{H}$ NMR (400 MHz, $\left.\mathrm{CDCl}_{3}\right): \delta(\mathrm{ppm}) 7.80\left(\mathrm{dd},{ }^{3} J_{\mathrm{H}-\mathrm{P}}=8.8 \mathrm{~Hz}, J_{\mathrm{H}-\mathrm{H}, \text { meta }}=2.2 \mathrm{~Hz}, 3 \mathrm{H}\right), 7.53\left(\mathrm{~d}, J_{\mathrm{H}-\mathrm{H}, \text { ortho }}=8.0 \mathrm{~Hz}, 3 \mathrm{H}\right)$, $7.23\left(\mathrm{dd}, J_{\mathrm{H}-\mathrm{H}, \text { ortho }}=8.0 \mathrm{~Hz}, J_{\mathrm{H}-\mathrm{H}, \text { meta }}=2.0 \mathrm{~Hz}, 3 \mathrm{H}\right), 1.28\left(\mathrm{~s}, 27 \mathrm{H}, \mathrm{C}\left(\mathrm{CH}_{3}\right)_{3}\right) ;{ }^{13} \mathrm{C} \mathrm{NMR}(101 \mathrm{MHz}, \mathrm{CDCl} 3): \delta(\mathrm{ppm}) 155.47(\mathrm{~d}$, $\left.{ }^{2} J_{\mathrm{C}-\mathrm{P}}=1.2 \mathrm{~Hz}\right), 148.01\left(\mathrm{~d},{ }^{1} J_{\mathrm{C}-\mathrm{P}}=10.8 \mathrm{~Hz}\right), 144.69\left(\mathrm{~d},{ }^{3} J_{\mathrm{C}-\mathrm{P}}=7.5 \mathrm{~Hz}\right), 129.88\left(\mathrm{~d},{ }^{2} J_{\mathrm{C}-\mathrm{P}}=34.4 \mathrm{~Hz}\right), 126.30\left(\mathrm{~d},{ }^{3} J_{\mathrm{C}-\mathrm{P}}=1.4 \mathrm{~Hz}\right)$, 125.62 (s), 34.73 (s, $\left.C\left(\mathrm{CH}_{3}\right)_{3}\right), 31.57\left(\mathrm{~s}, \mathrm{CH}_{3}\right) ;{ }^{31} \mathrm{P}\left({ }^{1} \mathrm{H}\right) \mathrm{NMR}\left(162 \mathrm{MHz}, \mathrm{CDCl}_{3}\right): \delta(\mathrm{ppm})-77.0$ (s). MS(EI): calcd m/z 441.26, found $\mathrm{m} / \mathrm{z} 441.3\left(\mathrm{M}^{+}\right)$. Anal. calcd (\%) for $\mathrm{C}_{30} \mathrm{H}_{36} \mathrm{NP}$ (441.60): C 81.60, H 8.22, N 3.17; found C 81.72, H 8.27, N 3.19 .

\section{Preparation of Complex 2, [(1)Rh(acac)(CO)]}

In a glovebox under nitrogen atmosphere, ligand 1 (0.017 g, $0.039 \mathrm{mmol})$ and (acetylacetonato)dicarbonylrhodium(I) [Rh(acac) $\left.(\mathrm{CO})_{2}\right](0.010 \mathrm{~g}, 0.039 \mathrm{mmol})$ were dissolved in toluene $(0.5 \mathrm{~mL})$; bubbles were observed immediately. The solution was layered with pentane $(5 \mathrm{~mL})$ and stored at $-35^{\circ} \mathrm{C}$ for 3 days. The solution was filtered and a yellow crystalline solid was collected. The solid was dried under vacuum for $12 \mathrm{~h}$ to afford the title complex $\left(0.015 \mathrm{~g},{ }^{58 \%}\right) .{ }^{1} \mathrm{H}$ NMR (400 MHz, C6 $\left.6 \mathrm{D}_{6}\right): \delta(\mathrm{ppm}) 8.70\left(\mathrm{dd},{ }^{3} J_{\mathrm{H}-\mathrm{P}}=12.4 \mathrm{~Hz}, J_{\mathrm{H}-\mathrm{H}, \text { meta }}=2.0 \mathrm{~Hz}, 3 \mathrm{H}\right), 7.69\left(\mathrm{dd}, J_{\mathrm{H}-\mathrm{H}, \text { ortho }}=8.0 \mathrm{~Hz},{ }^{4} J_{\mathrm{H}-\mathrm{P}}=2.4 \mathrm{~Hz}, 3 \mathrm{H}\right)$, $7.03\left(\mathrm{dd}, J_{\mathrm{H}-\mathrm{H}, \text { ortho }}=8.0 \mathrm{~Hz}, \mathrm{~J}_{\mathrm{H}-\mathrm{H}, \text { meta }}=2.4 \mathrm{~Hz}, 3 \mathrm{H}\right), 5.43(\mathrm{~s}, 1 \mathrm{H}, \mathrm{CH}, \mathrm{acac}), 2.00(\mathrm{~s}, 3 \mathrm{H}, \mathrm{CH}, \mathrm{acac}), 1.86(\mathrm{~s}, 3 \mathrm{H}, \mathrm{CH}, \mathrm{acac}), 1.08(\mathrm{~s}$, 27H, C(CH3)3); ${ }^{13} \mathrm{C}$ NMR (101 MHz, C $\left.6 \mathrm{D}_{6}\right): \delta(\mathrm{ppm}) 189.17\left(\mathrm{dd},{ }^{1} J_{\mathrm{C}-\mathrm{Rh}}=72.3 \mathrm{~Hz},{ }^{2} J_{\mathrm{C}-\mathrm{P}}=29.5 \mathrm{~Hz}, \mathrm{CO}\right), 188.34(\mathrm{~s}, \mathrm{CO}, \mathrm{acac}), 185.00$ (s, CO, acac), $154.78\left(\mathrm{~d},{ }^{2} J_{\mathrm{C}-\mathrm{P}}=1.9 \mathrm{~Hz}\right), 148.91\left(\mathrm{~d},{ }^{1} J_{\mathrm{C}-\mathrm{P}}=11.2 \mathrm{~Hz}\right), 141.49\left(\mathrm{~d},{ }^{2} J_{\mathrm{C}-\mathrm{P}}=45.3 \mathrm{~Hz}\right), 131.37\left(\mathrm{~d},{ }^{3} J_{\mathrm{C}-\mathrm{P}}=16.6 \mathrm{~Hz}\right), 127.44$

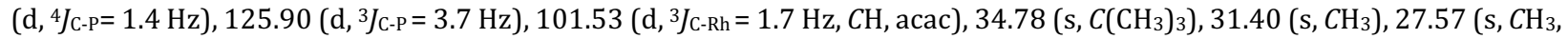
acac). ${ }^{31} \mathrm{P}\left({ }^{1} \mathrm{H}\right)$ NMR (162 MHz, $\left.\mathrm{C}_{6} \mathrm{D}_{6}\right): \delta(\mathrm{ppm})-1.28\left(\mathrm{~d},{ }^{1} J_{\mathrm{P}-\mathrm{Rh}}=189.0 \mathrm{~Hz}\right)$. IR: $v\left(\mathrm{~cm}^{-1}\right): 2961(\mathrm{w}), 1985(\mathrm{~s}, \mathrm{CO}), 1584(\mathrm{w})$, 1524(w), 1474(s), 1391(w), 1051 (s), 729(s). Anal. calcd (\%) for $\mathrm{C}_{36} \mathrm{H}_{43} \mathrm{NO}_{3} \mathrm{PRh}$ (671.62): C 64.38, $\mathrm{H}$ 6.45, N 2.09; found C 64.37, H 7.04, N 1.90 .

\section{Selected Spectra}

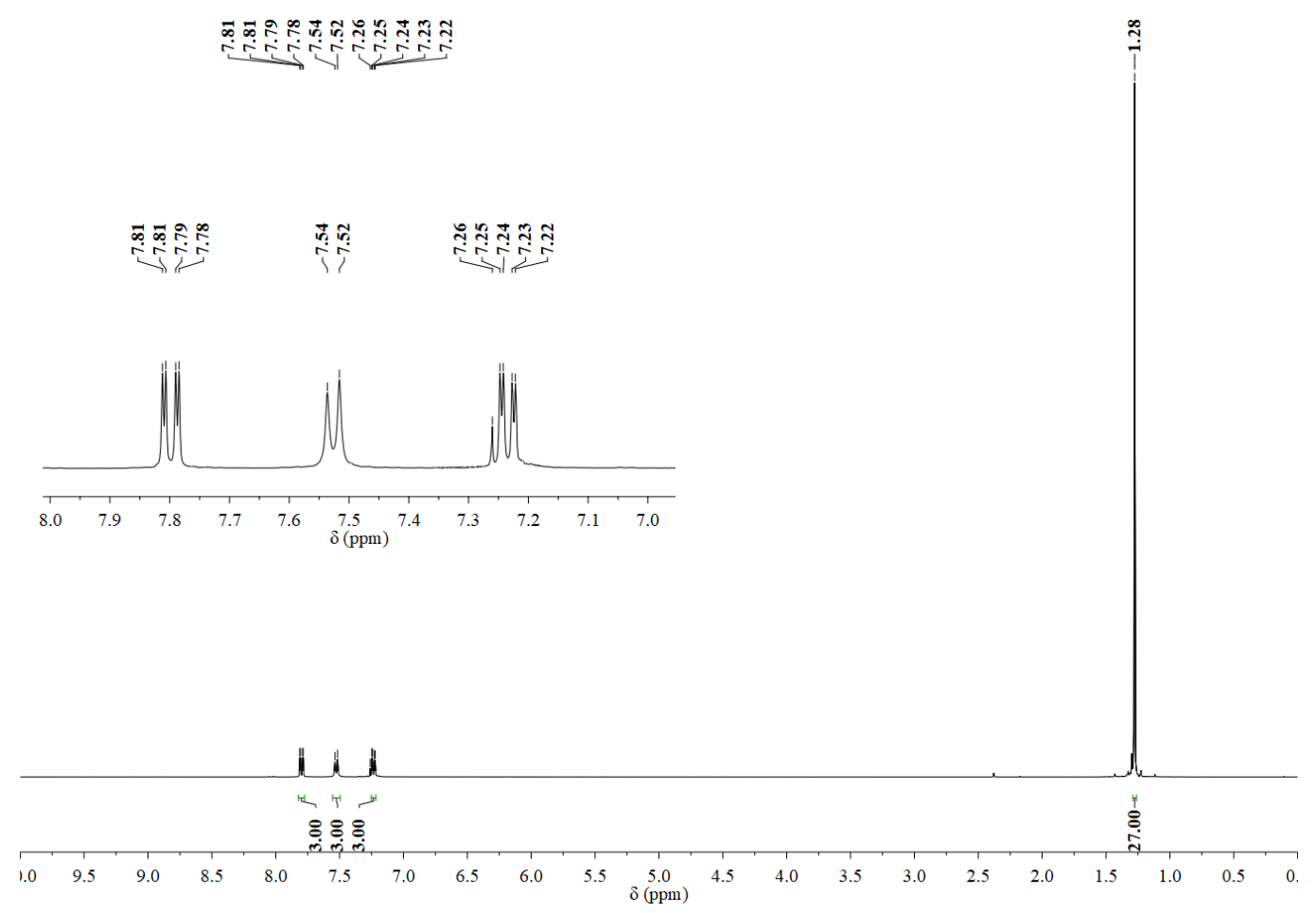

Figure S1. ${ }^{1} \mathrm{H}$ NMR spectrum of 1 in $\mathrm{CDCl}_{3}$ solution. 


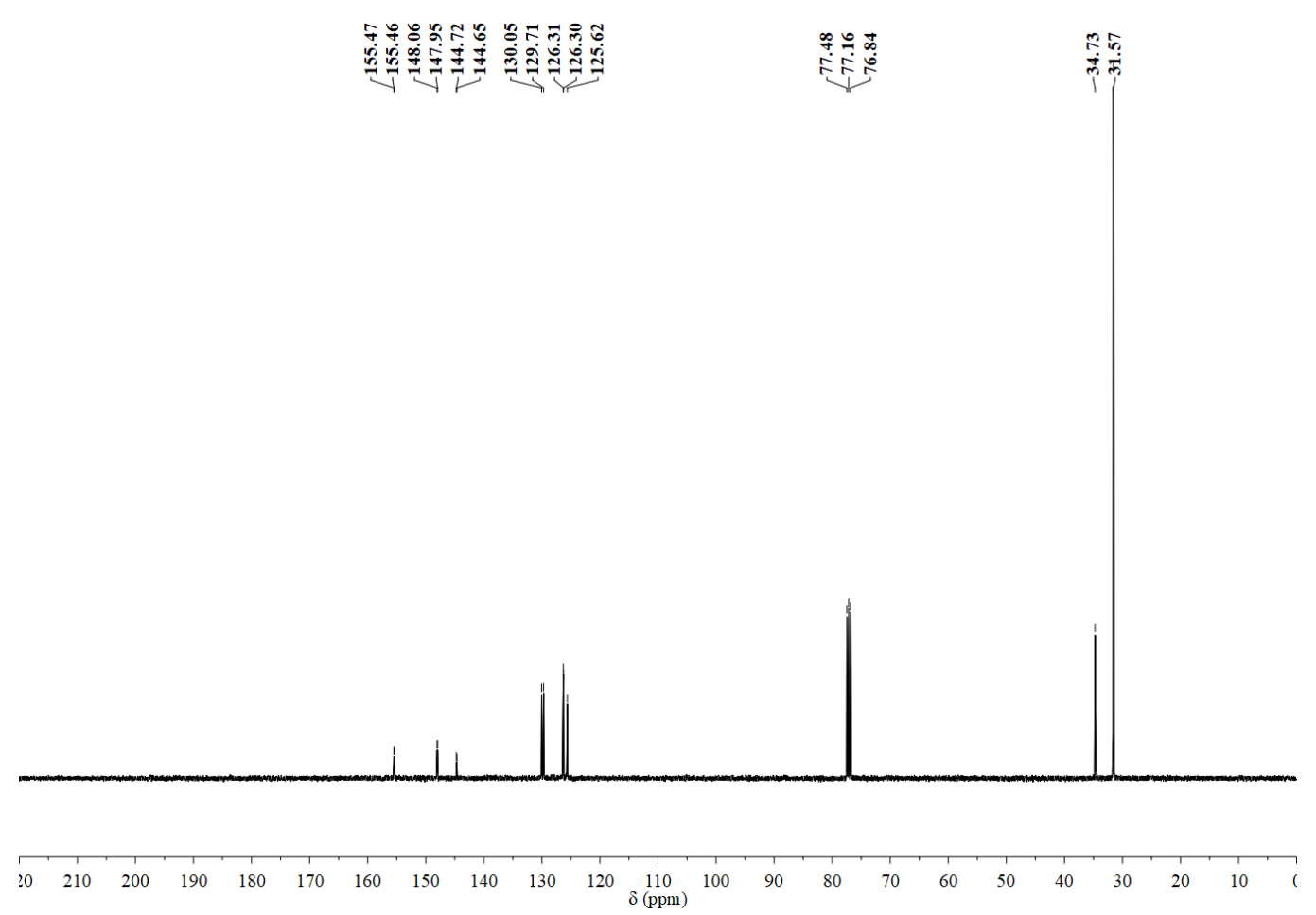

Figure S2. ${ }^{13} \mathrm{C}$ NMR spectrum of $\mathbf{1}$ in $\mathrm{CDCl}_{3}$ solution.

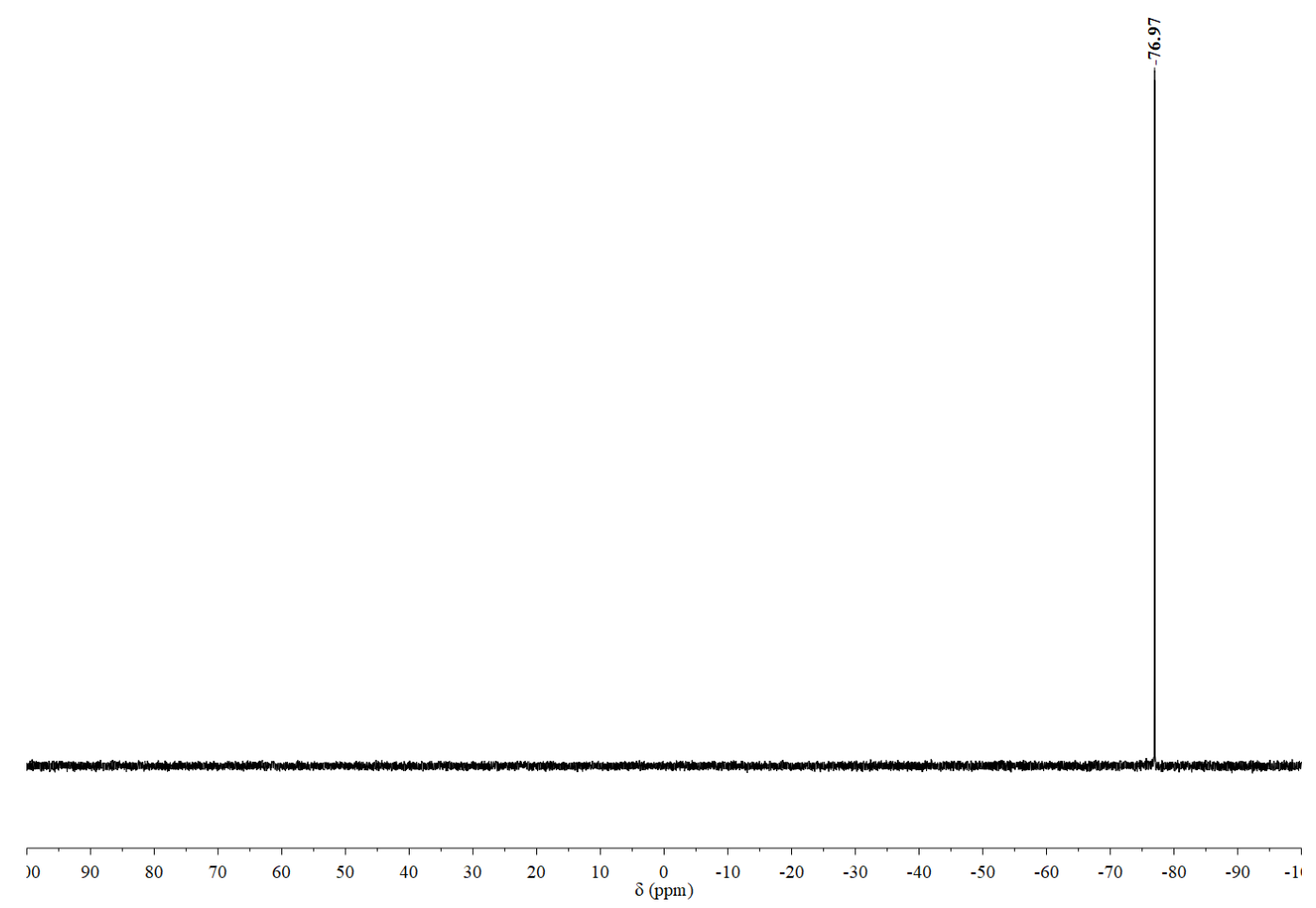

Figure S3. ${ }^{31} \mathrm{P}$ NMR spectrum of 1 in $\mathrm{CDCl}_{3}$ solution. 


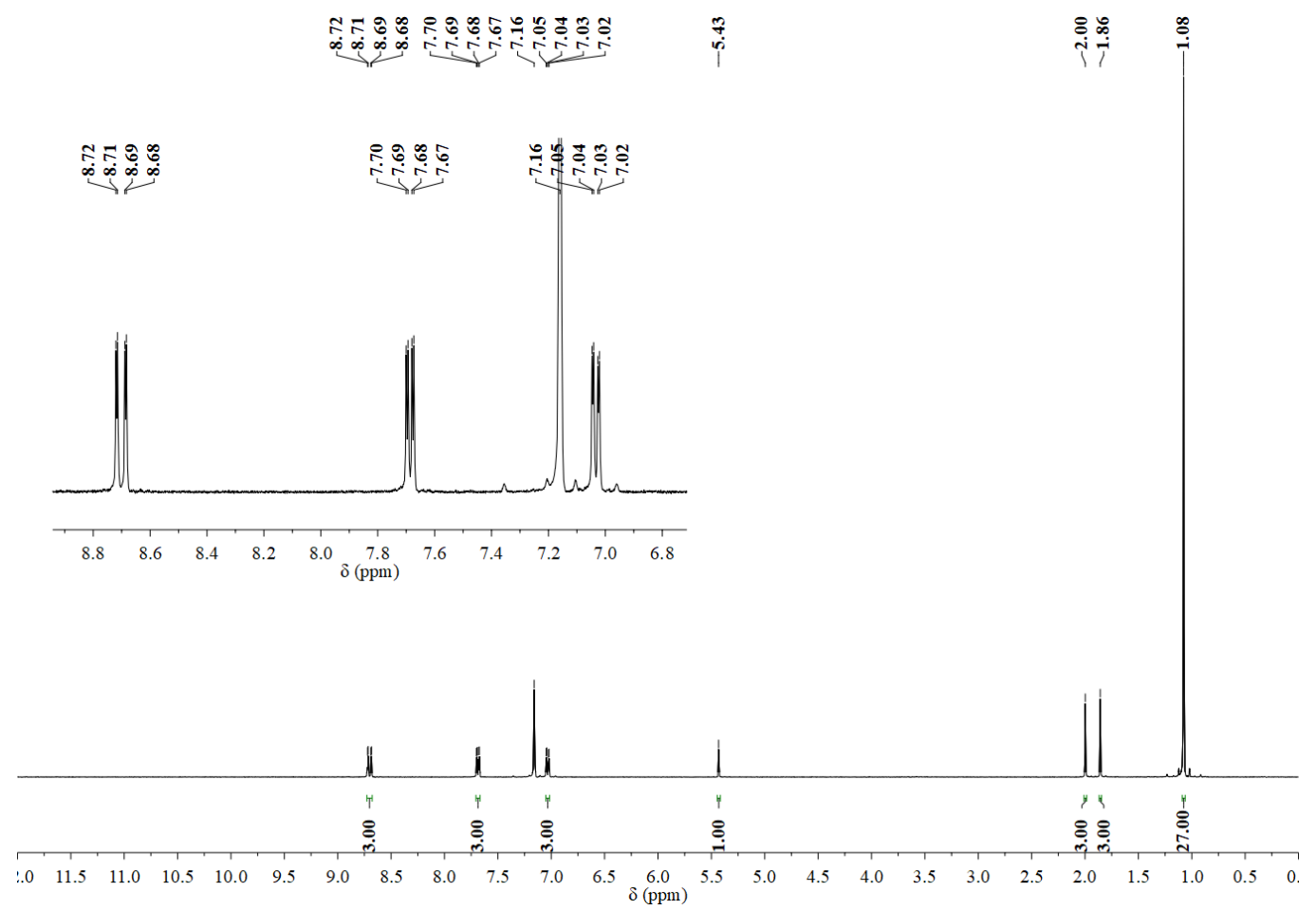

Figure S4. ${ }^{1} \mathrm{H}$ NMR spectrum of $\mathbf{2}$ in $\mathrm{C}_{6} \mathrm{D}_{6}$ solution.

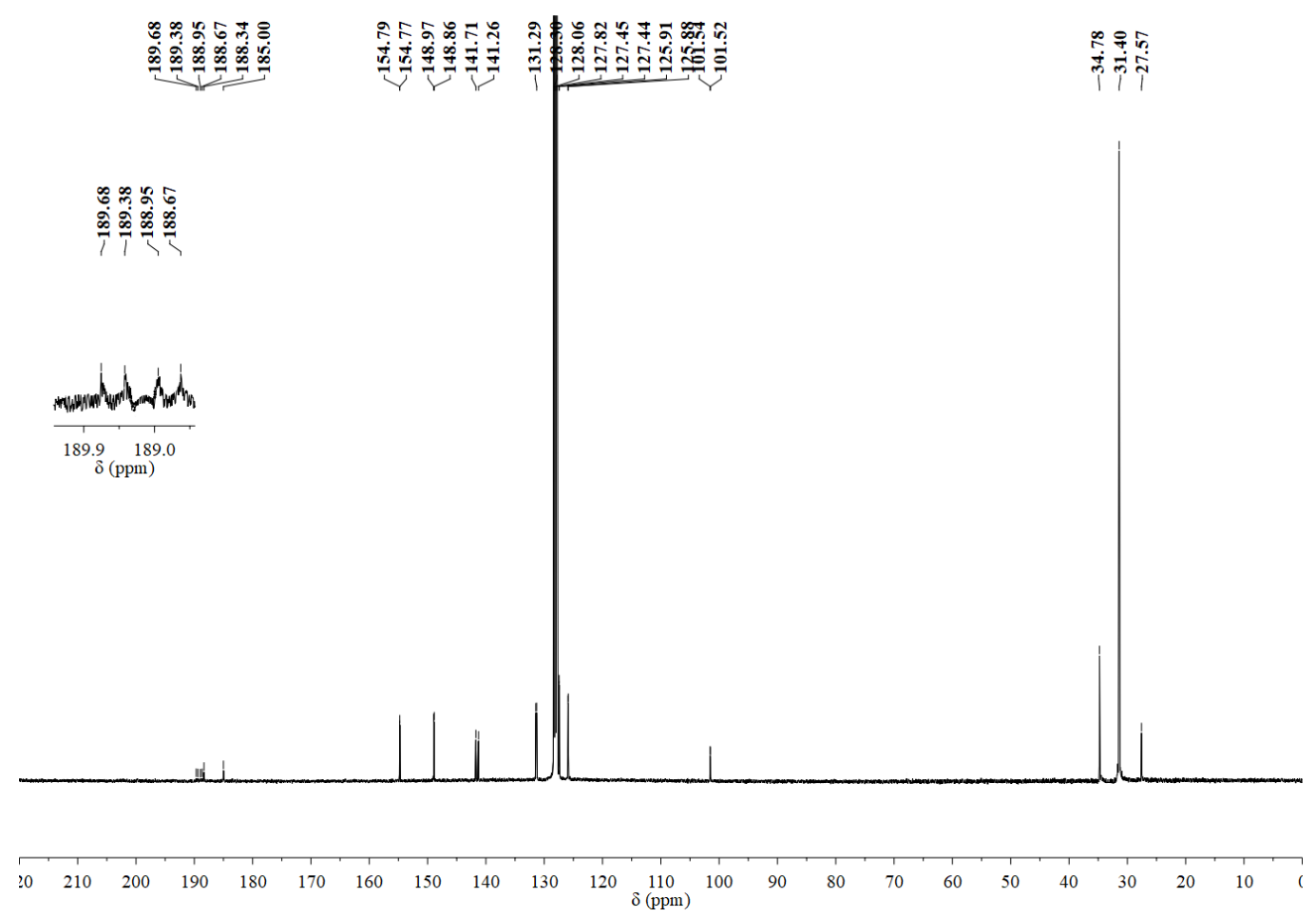

Figure S5. ${ }^{13} \mathrm{C}$ NMR spectrum of 2 in $\mathrm{C}_{6} \mathrm{D}_{6}$ solution. 


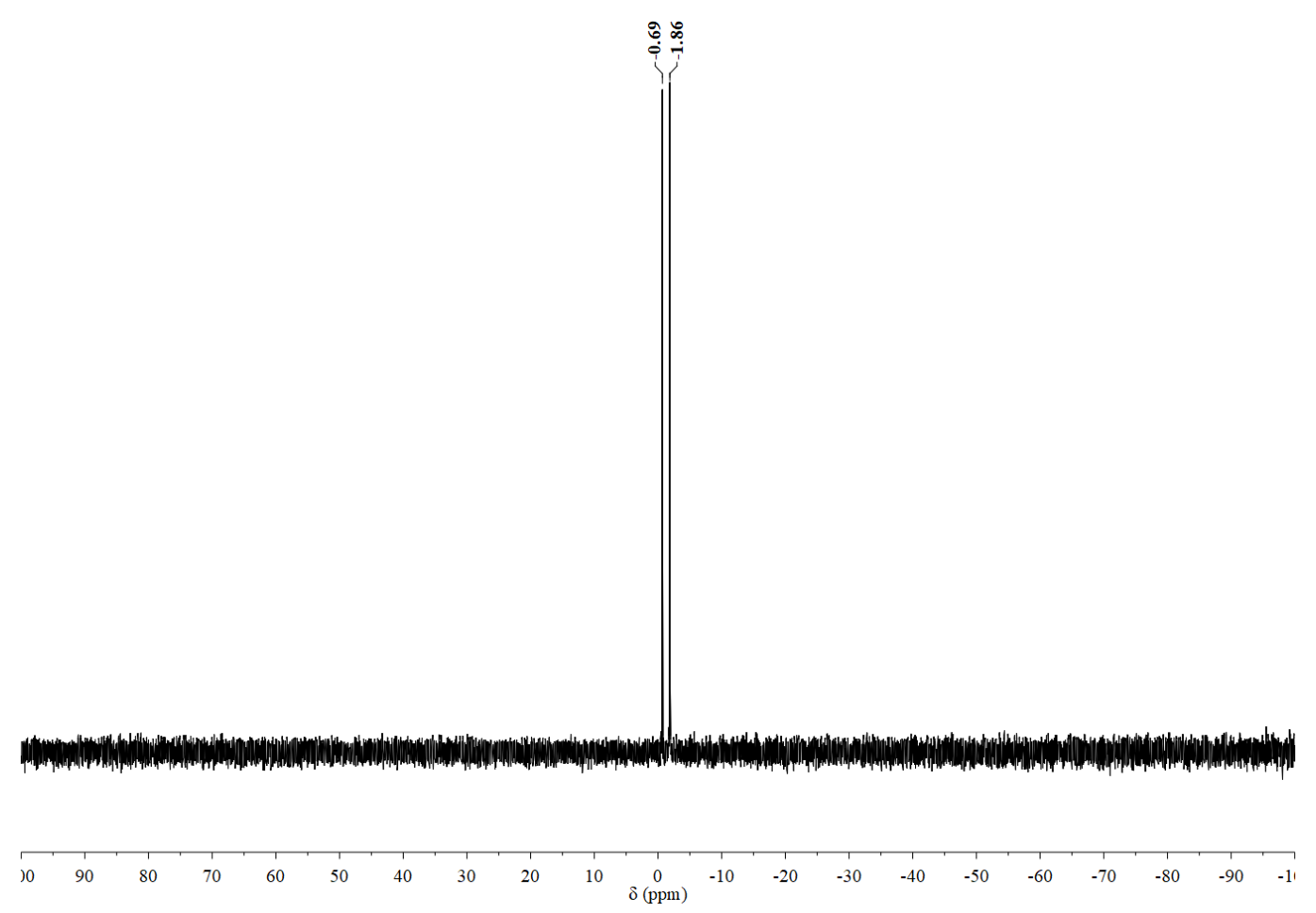

Figure S6. ${ }^{31} \mathrm{P}$ NMR spectrum of 2 in $\mathrm{C}_{6} \mathrm{D}_{6}$ solution.

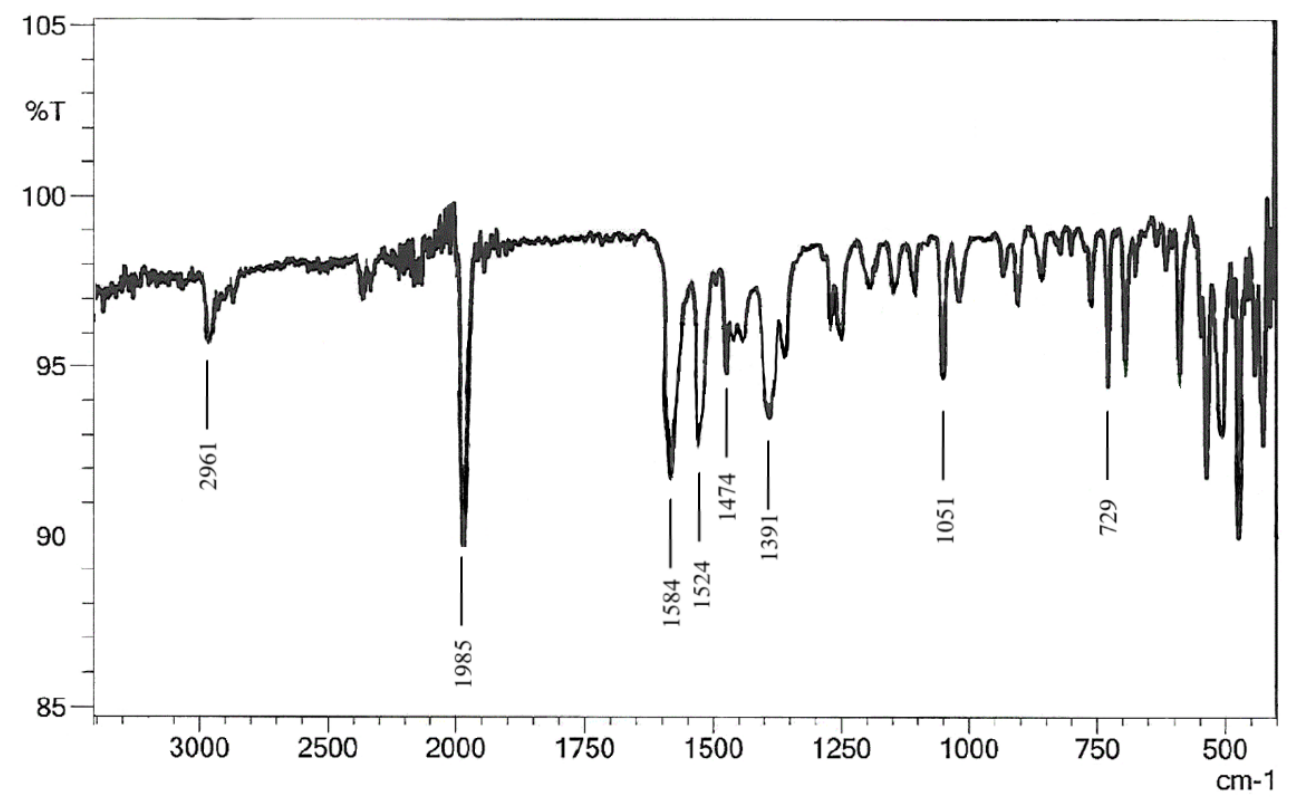

Figure S7. IR spectrum of 2.

\section{General Procedure for Hydroformylation}

Ligand 1, $\left[\mathrm{Rh}(\mathrm{acac})(\mathrm{CO})_{2}\right]$ and toluene were mixed in a glovebox and transferred via gastight syringe into a previously dried 300-mL stainless steel Parr reactor. The reactor was purged twice with $\mathrm{N}_{2}$ ( 7 bar), then three times with syngas ( 7 bar). Substrate was added into the Parr reactor via another gastight syringe, then the reactor was purged twice more with syngas ( 7 bar), and released to 1 bar. The reactor was then heated to the desired working 
temperature, a continuous working pressure of syngas was established, and stirring was initiated. The reaction mixture was stirred for the desired reaction time, then agitation was stopped and the reactor was cooled to room temperature in an ice bath. The gas inside the Parr reactor was slowly vented in the hood to a $4.0 \mathrm{~mL}$ cold bath of toluene $\left(0^{\circ} \mathrm{C}\right)$. The reactor was opened and the organic phase was diluted to a defined volume. Aliquots taken from the organic phase and from cold toluene were analyzed by GC-FID. Amounts of starting substrates and products were determined by analysis of both the organic phase and the toluene solution for gas venting, based on predetermined calibration curves. Aldehyde TOF (turnover frequency) was calculated from the molar quantity of aldehyde formed per mole of $\mathrm{Rh}$ (acac) $(\mathrm{CO})_{2}$ precatalyst per hour of reaction time.

Hydroformylation experiments of 2,3-dihydrofuran and 3,4-dihydro-2H-pyran were reproduced from literature reports under their reported conditions. ${ }^{3-4}$ The hydroformylation products were determined by GC-MS and GC-FID and isomer aldehydes were identified by different retention times on GC based on boiling points. None of the experiments produced hydrogenation products.

\section{Hydroformylation of 1-Octene}

Hydroformylation experiments of 1 -octen ${ }^{5}$ were conducted by dissolving catalyst precursors in toluene in the autoclave, then pressurizing with syngas and heating up to the desired pressure and temperature over 40 min. 1-Octene was added under pressure, and the reactions run for $1 \mathrm{~h}$. To compare results in our laboratory to those of experiments reported using the same reaction conditions, catalyst precursor and 1-octene were dissolved in toluene in the autoclave, then the desired pressure of syngas was added and the mixture was heated over 20 min to the desired temperature, followed by a reaction time of $1 \mathrm{~h}$. The results are summarized in Table S1.

Table S1. Hydroformylation of 1-octene. ${ }^{a}$

\begin{tabular}{|c|c|c|c|c|c|c|}
\hline Entry & $\mathrm{L}$ & $\begin{array}{l}\mathrm{L} / \mathrm{M} \\
\text { ratio }\end{array}$ & $\begin{array}{c}\text { Alkene } \\
\text { conv } \\
(\%)\end{array}$ & $\begin{array}{c}\text { Aldehyde } \\
(\%)\end{array}$ & $\begin{array}{l}l: b \\
\text { ratio }\end{array}$ & $\begin{array}{l}\text { Aldehyde } \\
\text { TOF }\left(\mathrm{h}^{-1}\right)\end{array}$ \\
\hline 1 & $\mathrm{PPh}_{3}$ & 20 & 42 & 42 & 2.8 & $2100^{5}$ \\
\hline 2 & $\mathrm{PPh}_{3}$ & 20 & 76 & 28 & 2.8 & 1390 \\
\hline 3 & 1 & 20 & 1 & 0.3 & $-b$ & 15 \\
\hline 4 & 1 & 4 & 80 & 56 & 3.1 & 2780 \\
\hline $5^{c}$ & 1 & 2 & 99 & 53 & 1.5 & 5300 \\
\hline$\sigma^{c, d}$ & 1 & 2 & 20 & 13 & 3.2 & 1270 \\
\hline
\end{tabular}

$a\left[\mathrm{Rh}(\mathrm{acac})(\mathrm{CO})_{2}\right], 0.020 \mathrm{~mol} \%$; [1-octene $]_{\text {initial }}=0.84 \mathrm{M}$ in toluene; $\mathrm{H}_{2} / \mathrm{CO}(1: 1) 20$ bar. Unless noted otherwise, $80{ }^{\circ} \mathrm{C}, 1 \mathrm{~h} .{ }^{b}$ Only $n$-aldehyde detected. $c$ Reaction time 0.5 h. ${ }^{d}$ Reaction temp $60{ }^{\circ} \mathrm{C}$.

\section{X-ray Diffraction Studies for Complex 2, [(1)Rh(acac)(C0)]}

Experimental.

Single crystals of $\mathbf{2}$ were recrystallized from a mixture of THF and pentane by vapor diffusion. A suitable crystal was selected and mounted on a loop with Paratone oil a Bruker D8 Venture diffractometer. The crystal was kept at 100(2) K during data collection. Using Olex2, ${ }^{6}$ the structure was solved with the SHELXT7 ${ }^{7}$ structure solution program using Intrinsic Phasing, and refined with the SHELXL ${ }^{8}$ refinement package using Least Squares minimization.

Crystal Data.

$\mathrm{C}_{72} \mathrm{H}_{86} \mathrm{~N}_{2} \mathrm{O}_{6} \mathrm{P}_{2} \mathrm{Rh}_{2}(M=1343.19 \mathrm{~g} / \mathrm{mol}):$ triclinic, space group P-1 (no. 2), $a=10.6289(5) \AA, b=10.8230(5) \AA, c=$ $17.8290(8) \AA, \alpha=84.254(2)^{\circ}, \beta=78.440(2)^{\circ}, \gamma=63.6990(10)^{\circ}, V=1801.22(15) \AA^{3}, Z=1, T=100(2) \mathrm{K}, \mu(\mathrm{MoK} \alpha)=$ $0.551 \mathrm{~mm}^{-1}, \mathrm{D}_{\text {calc }}=1.238 \mathrm{~g} / \mathrm{cm}^{3}, 16475$ reflections measured $\left(4.342^{\circ} \leq 2 \Theta \leq 56.564^{\circ}\right), 8804$ unique $\left(R_{\text {int }}=0.0260\right.$, $\left.\mathrm{R}_{\text {sigma }}=0.0454\right)$ which were used in all calculations. The final $R_{1}$ was $0.0461\left(\mathrm{I}>2 \sigma(\mathrm{I})\right.$ ) and $w R_{2}$ was 0.1130 (all data). 
Table S2. Crystal data and structure refinement for $\mathbf{2}$.

\begin{tabular}{|c|c|}
\hline Empirical formula & $\mathrm{C}_{72} \mathrm{H}_{86} \mathrm{~N}_{2} \mathrm{O}_{6} \mathrm{P}_{2} \mathrm{Rh}_{2}$ \\
\hline Formula weight & 1343.19 \\
\hline Temperature/K & $100(2)$ \\
\hline Crystal system & triclinic \\
\hline Space group & $\mathrm{P}-1$ \\
\hline $\mathbf{a} / \AA ̊ \AA$ & $10.6289(5)$ \\
\hline b/Å & $10.8230(5)$ \\
\hline c/Å & $17.8290(8)$ \\
\hline$\alpha /{ }^{\circ}$ & $84.254(2)$ \\
\hline$\beta /{ }^{\circ}$ & $78.440(2)$ \\
\hline$\gamma /{ }^{\circ}$ & $63.6990(10)$ \\
\hline Volume $/ \AA^{3}$ & $1801.22(15)$ \\
\hline $\mathbf{Z}$ & 1 \\
\hline$\rho_{\text {calcg }} / \mathrm{cm}^{3}$ & 1.238 \\
\hline $\boldsymbol{\mu} / \mathbf{m m}^{-1}$ & 0.551 \\
\hline$F(000)$ & 700.0 \\
\hline Crystal size $/ \mathrm{mm}^{3}$ & $0.254 \times 0.119 \times 0.113$ \\
\hline Radiation & $\operatorname{MoK} \alpha(\lambda=0.71073)$ \\
\hline $2 \Theta$ range for data collection $/{ }^{\circ}$ & 4.342 to 56.564 \\
\hline Index ranges & $-14 \leq \mathrm{h} \leq 10,-14 \leq \mathrm{k} \leq 13,-23 \leq \mathrm{l} \leq 23$ \\
\hline Reflections collected & 16475 \\
\hline Independent reflections & $8804\left[R_{\text {int }}=0.0260, R_{\text {sigma }}=0.0454\right]$ \\
\hline Data/restraints/parameters & $8804 / 378 / 390$ \\
\hline Goodness-of-fit on $F^{2}$ & 1.206 \\
\hline Final $R$ indexes $[I>=2 \sigma(I)]$ & $\mathrm{R}_{1}=0.0461, \mathrm{w} \mathrm{R}_{2}=0.0909$ \\
\hline Final $R$ indexes [all data] & $\mathrm{R}_{1}=0.0618, \mathrm{wR}_{2}=0.1130$ \\
\hline Largest diff. peak/hole / e $\AA^{-3}$ & $1.01 /-0.76$ \\
\hline
\end{tabular}


Table S3. Bond lengths in $\AA$ for 2.

\begin{tabular}{|c|c|c|c|c|c|}
\hline Atom & Atom & Length/Å & Atom & Atom & Length/Å \\
\hline Rh1 & P1 & $2.2080(8)$ & $\mathrm{C} 24$ & $\mathrm{C} 23$ & $1.375(4)$ \\
\hline Rh1 & 03 & $2.044(2)$ & C12 & C17 & $1.374(4)$ \\
\hline Rh1 & 02 & $2.067(2)$ & $\mathrm{C} 23$ & $\mathrm{C} 22$ & $1.405(4)$ \\
\hline Rh1 & C1 & $1.821(3)$ & C14 & C15 & $1.403(4)$ \\
\hline P1 & $\mathrm{C} 2$ & $1.832(3)$ & C8 & C6 & $1.530(4)$ \\
\hline P1 & C13 & $1.834(3)$ & C8 & C11 & $1.548(5)$ \\
\hline P1 & $\mathrm{C} 22$ & $1.823(3)$ & C8 & C9 & $1.523(5)$ \\
\hline 03 & C32 & $1.288(4)$ & C8 & C10 & $1.529(5)$ \\
\hline 02 & C34 & $1.249(4)$ & $\mathrm{C} 5$ & C6 & $1.390(4)$ \\
\hline 01 & C1 & $1.142(4)$ & C33 & C32 & $1.396(4)$ \\
\hline N1 & $\mathrm{C} 12$ & $1.466(4)$ & C33 & C34 & $1.399(5)$ \\
\hline N1 & C3 & $1.460(4)$ & $\mathrm{C} 26$ & $\mathrm{C} 28$ & $1.527(4)$ \\
\hline N1 & $\mathrm{C} 23$ & $1.467(3)$ & C32 & C35 & $1.512(5)$ \\
\hline $\mathrm{C} 2$ & $\mathrm{C} 7$ & $1.398(4)$ & C15 & C16 & $1.399(4)$ \\
\hline $\mathrm{C} 2$ & C3 & $1.398(4)$ & C15 & C18 & $1.531(5)$ \\
\hline C7 & C6 & $1.412(4)$ & C31 & $\mathrm{C} 28$ & $1.536(5)$ \\
\hline C13 & $\mathrm{C} 12$ & $1.393(4)$ & $\mathrm{C} 28$ & $\mathrm{C} 29$ & $1.529(4)$ \\
\hline C13 & $\mathrm{C} 14$ & $1.386(4)$ & $\mathrm{C} 28$ & C30 & $1.532(5)$ \\
\hline $\mathrm{C} 27$ & $\mathrm{C} 22$ & $1.397(4)$ & C34 & C36 & $1.503(4)$ \\
\hline $\mathrm{C} 27$ & $\mathrm{C} 26$ & $1.401(4)$ & C16 & $\mathrm{C} 17$ & $1.390(4)$ \\
\hline C25 & $\mathrm{C} 24$ & $1.399(4)$ & C18 & C19 & $1.535(5)$ \\
\hline C25 & $\mathrm{C} 26$ & $1.396(4)$ & C18 & $\mathrm{C} 20$ & $1.529(6)$ \\
\hline $\mathrm{C} 4$ & C3 & $1.382(4)$ & C18 & $\mathrm{C} 21$ & $1.531(5)$ \\
\hline $\mathrm{C} 4$ & C5 & $1.387(4)$ & & & \\
\hline
\end{tabular}

Table S4. Bond angles for 2.

\begin{tabular}{|llllllll|}
\hline Atom & Atom & Atom & Angle $^{\circ}$ & Atom & Atom & Atom & Angle $/^{\circ}$ \\
O3 & Rh1 & P1 & $94.54(6)$ & C9 & C8 & C11 & $107.7(3)$ \\
O3 & Rh1 & O2 & $89.26(9)$ & C9 & C8 & C10 & $109.9(3)$ \\
O2 & Rh1 & P1 & $175.79(7)$ & C10 & C8 & C6 & $108.5(3)$ \\
\hline
\end{tabular}




\begin{tabular}{|c|c|c|c|c|c|c|c|}
\hline $\mathrm{C} 1$ & Rh1 & P1 & $86.43(10)$ & C10 & C8 & C11 & $108.7(3)$ \\
\hline $\mathrm{C} 1$ & Rh1 & 03 & $178.88(12)$ & $\mathrm{C} 4$ & C5 & C6 & $121.9(3)$ \\
\hline C1 & Rh1 & 02 & 89.78(11) & C7 & C6 & C8 & $119.4(3)$ \\
\hline $\mathrm{C} 2$ & P1 & Rh1 & $126.62(10)$ & C5 & C6 & C7 & 118.3(3) \\
\hline $\mathrm{C} 2$ & P1 & C13 & $95.00(13)$ & C5 & C6 & C8 & $122.3(3)$ \\
\hline C13 & P1 & Rh1 & 119.44(10) & C32 & C33 & C34 & $126.3(3)$ \\
\hline $\mathrm{C} 22$ & P1 & Rh1 & $118.48(10)$ & C27 & $\mathrm{C} 22$ & P1 & $126.3(2)$ \\
\hline $\mathrm{C} 22$ & P1 & $\mathrm{C} 2$ & $95.14(13)$ & $\mathrm{C} 27$ & C22 & $\mathrm{C} 23$ & $119.5(3)$ \\
\hline $\mathrm{C} 22$ & P1 & C13 & 95.13(13) & $\mathrm{C} 23$ & C22 & P1 & $114.2(2)$ \\
\hline C32 & 03 & Rh1 & $126.2(2)$ & C27 & C26 & $\mathrm{C} 28$ & $119.6(3)$ \\
\hline C34 & 02 & Rh1 & $126.6(2)$ & C25 & C26 & $\mathrm{C} 27$ & $117.5(3)$ \\
\hline C12 & N1 & $\mathrm{C} 23$ & $108.9(2)$ & $\mathrm{C} 25$ & C26 & $\mathrm{C} 28$ & $122.9(3)$ \\
\hline C3 & N1 & C12 & $107.5(2)$ & 03 & C32 & C33 & $125.6(3)$ \\
\hline C3 & N1 & $\mathrm{C} 23$ & $108.0(2)$ & 03 & C32 & C35 & $115.4(3)$ \\
\hline C7 & C2 & P1 & $126.6(2)$ & C33 & C32 & C35 & $119.0(3)$ \\
\hline C7 & $\mathrm{C} 2$ & C3 & $120.0(3)$ & C14 & C15 & C18 & $120.1(3)$ \\
\hline C3 & C2 & P1 & $113.3(2)$ & C16 & C15 & C14 & $116.9(3)$ \\
\hline C2 & C7 & C6 & $120.0(3)$ & C16 & C15 & C18 & $123.0(3)$ \\
\hline C12 & C13 & P1 & $113.9(2)$ & 01 & $\mathrm{C} 1$ & Rh1 & $178.5(3)$ \\
\hline C14 & C13 & P1 & 126.1(2) & C26 & C28 & C31 & 109.1(3) \\
\hline C14 & C13 & C12 & $120.0(3)$ & C26 & C28 & C29 & $112.0(3)$ \\
\hline $\mathrm{C} 22$ & $\mathrm{C} 27$ & C26 & $121.2(3)$ & C26 & $\mathrm{C} 28$ & C30 & $109.7(3)$ \\
\hline C26 & C25 & C24 & $122.2(3)$ & C29 & C28 & C31 & $108.9(3)$ \\
\hline C3 & C4 & C5 & $119.6(3)$ & C29 & C28 & C30 & $108.0(3)$ \\
\hline $\mathrm{C} 23$ & C24 & C25 & 119.3(3) & C30 & C28 & C31 & $109.2(3)$ \\
\hline C13 & C12 & N1 & 118.6(3) & 02 & C34 & C33 & $126.0(3)$ \\
\hline C17 & C12 & N1 & $121.2(3)$ & 02 & C34 & C36 & 116.1(3) \\
\hline C17 & C12 & C13 & $120.2(3)$ & C33 & C34 & C36 & $117.9(3)$ \\
\hline $\mathrm{C} 2$ & C3 & N1 & $119.2(2)$ & C17 & C16 & C15 & $122.4(3)$ \\
\hline C4 & C3 & N1 & $120.6(3)$ & C12 & C17 & C16 & $119.2(3)$ \\
\hline C4 & C3 & $\mathrm{C} 2$ & $120.2(3)$ & C15 & C18 & C19 & 108.3(3) \\
\hline C24 & $\mathrm{C} 23$ & N1 & $121.7(2)$ & C20 & C18 & C15 & $110.2(3)$ \\
\hline C24 & $\mathrm{C} 23$ & $\mathrm{C} 22$ & $120.3(3)$ & $\mathrm{C} 20$ & C18 & C19 & 109.3(3) \\
\hline
\end{tabular}




\begin{tabular}{|llllllll|}
\hline C22 & C23 & N1 & $118.0(2)$ & C20 & C18 & C21 & $108.5(3)$ \\
C13 & C14 & C15 & $121.2(3)$ & C21 & C18 & C15 & $112.3(3)$ \\
C6 & C8 & C11 & $109.6(3)$ & C21 & C18 & C19 & $108.1(3)$ \\
C9 & C8 & C6 & $112.4(3)$ & & & & \\
\hline
\end{tabular}

\section{References}

1. Michinobu, T.; Tsuchida, E.; Nishide, H. "3,4'-Bis[bis(t-butyl- and methoxy-phenyl)amino]stilbene Bis(cation radical)s and Their Electrochemical and Magnetic Properties." Bull. Chem. Soc. Jpn. 2000, 73, 1021-1027.

2. Talipov, M. R.; Hossain, M. M.; Boddeda, A.; Thakur, K.; Rathore, R. "A search for blues brothers: X-ray crystallographic/spectroscopic characterization of the tetraarylbenzidine cation radical as a product of aging of solid magic blue." Org. Biomol. Chem. 2016, 14, 2961-2968.

3. Polo, A.; Claver, C.; Castillón, S.; Ruiz, A.; Bayón, J. C.; Real, J.; Mealli, C.; Masi, D. "Regioselective hydroformylation of cyclic vinyl and allyl ethers with rhodium catalysts. Crucial influence of the size of the phosphorus cocatalyst."Organometallics 1992, 11, 3525-3533.

4. Polo, A.; Real, J.; Claver, C.; Castillón, S.; Bayón, J. C. "Low-pressure selective hydroformylation of 2,3- and 2,5dihydrofuran with a rhodium catalyst. Unexpected influence of the auxiliary ligand tris(o-t-butylphenyl) phosphite." J. Chem. Soc., Chem. Commun. 1990, 600-601.

5. Buhling, A.; Kamer, P. C. J.; van Leeuwen, P. W. N. M. "Rhodium catalysed hydroformylation of higher alkenes using amphiphilic ligands." J. Mol. Catal. A: Chem. 1995, 98, 69-80.

6. Dolomanov, O. V.; Bourhis, L. J.; Gildea, R. J.; Howard, J. A. K.; Puschmann, H. "OLEX2: a complete structure solution, refinement and analysis program." J. Appl. Cryst. 2009, 42, 339-341.

7. Sheldrick, G. M. "SHELXT - Integrated space-group and crystal-structure determination." Acta Cryst. 2015, A71, 3-8.

8. Sheldrick, G. M. "Crystal Refinement with SHELXL." Acta Cryst. 2015, C71, 3-8. 